\title{
Diseño de estrategias de mercadeo para el programa de ingeniería de Materiales de una universidad del Caribe colombiano: Referentes de demanda potencial colegios en Barranquilla, Soledad, Baranoa y Piojón \\ Design of marketing strategies for a university in the colombian Caribbean materials engineering programme: potential demand concerning schools in Barranquilla, Soledad, Baranoa and Piojó
}

\author{
Winston Fontalvo Cerpa \\ Msc. Ingeniero Industrial, Docente Programa Ingeniería Industrial Universidad Autónoma del Caribe, winston.fontalvo@uac.edu.co
}

Beatriz María Cardozo Arrieta

Msc (c). Ingeniera de materiales, Docente del Programa Ingeniería de Materiales Universidad Autónoma del Caribe, bcardozo@uac.edu.co

\section{RESUMEN}

El objetivo de este trabajo es realizar un estudio del mercado del programa de ingeniería de materiales, debido a la poca participación de la comunidad estudiantil activa frente a otras ingenierías que tienen mayo acogida, observándose un comportamiento de bajo número de las matrículas frente a otros programas de ingeniería, empleando para ello una serie de encuestas a 885 estudiantes de $11^{\circ}$ grado de los diversos establecimientos educativos del área metropolitana de Barranquilla, 20 públicos y 22 privados, con el fin de conocer las preferencias por programas académicos y saber que tanta información poseen los estudiantes de secundaria acerca de esta carrera y otra encuesta a 17 estudiantes del programa de Ing. de Materiales (a quienes se denominó cliente interno). Con base en los resultados arrojados en la encuesta se diseñaron estrategias que permitan incrementar el reconocimiento y la percepción de la ingeniería de materiales, así como su impacto en la sociedad, especificando el plan de acción y el presupuesto para su implementación.

Palabras Clave: ingeniería de materiales, percepción, mercadeo, estrategias, impacto social.

\begin{abstract}
This paper purpose is to make a study of materials engineering program's market, due to low participation of student community active against other engineering that have more reception, observed behavior of low number of enrollments compared to other engineering programs, employing a series of surveys of students of various educational institutions in the metropolitan area of Barranquilla, 20 public and 22 private, in order to meet the preferences for academic programs and know that much information possessed by high school students this career and other survey to 17 students of Materials Engineering program (who are called internal customer). Based on the results obtained in the survey were designed strategies to increase the recognition and perception of material engineering materials and its impact on society, specifying the action plan and budget for implementation.
\end{abstract}

Key words: Materials engineering, perception, marketing, strategies, social impact.

\section{Introducción}

La Universidad Autónoma del Caribe, en su afán de expandir sus horizontes académicos, y proyectarse como la mejor opción a la hora de estudiar una carrera de formación profesional y/o técnica, expande su oferta académica en la Facultad de Ingeniería, abriendo nuevos caminos con la Ingeniería de materiales, la cual inicia actividades académicas en el primer período académico de 2011, siendo a nivel regional, la única Universidad que ofrece este programa. 
Sin embargo, el comportamiento de las matrículas en el programa ha sido considerablemente bajo, lo que ocasiona bajos ingresos y potencial de autosostenibilidad, razón por la cual se hace necesario analizar la situación presentada, para ofrecer alternativas de solución que permitan incrementar la población estudiantil en Ingeniería de Materiales.

El presente trabajo presenta precisamente el análisis mencionado, así como las propuestas para mejorar la situación del programa. Identifica, inicialmente, las características generales a través de un análisis estratégico en el que se incluye en el primer capítulo el análisis de la situación externa que involucra los aspectos geográficos, demográficos, de oferta y demanda relacionadas con el programa.

En segunda instancia se analiza la situación interna respecto a la ingeniería de materiales, generalizando las características de la oferta de la Institución, y concluyendo con el análisis de una encuesta aplicada a los estudiantes del programa. Posteriormente se presenta el análisis DOFA de la Ingeniería de Materiales de acuerdo con los resultados presentados en los dos numerales anteriores.

Finalmente, se esbozan acciones propuestas de marketing, concentradas en estrategias de comunicación, incluyendo el cronograma de ejecución y el presupuesto de implementación del plan propuesto, así como los ingresos estimados en las acciones que puedan generarlos de manera directa.

\section{Referentes Teóricos}

El marketing es un componente esencial en la gestión de cualquier organización, sin distingo del sector en que se desenvuelva, máxime en las condiciones actuales. Siguiendo a Kotler (2001) el marketing es un proceso a través del cual los individuos y grupos satisfacen sus necesidades mediante la negociación y el intercambio, condición que se persigue en cualquier actividad económica.

En el caso de los servicios, por sus características especiales, exigen un marketing diferente (Grande, 2005), que permita una mejor comprensión por parte del mercado objetivo y que, al mismo tiempo, lo cautive para lograr el acercamiento que lleve al intercambio.

Un servicio es un fenómeno complicado, ello se debe a su naturaleza intangible, que se traduce en dificultad para el cliente al evaluarlo, al mismo tiempo que es difícil aplicar control de calidad, como también el marketing tradicional a los servicios (Grönroos, 1990). “Sin embargo, cabe aquí preguntarse: ¿qué queda del cliente? y ¿cómo se pueden resolver las necesidades que ese mismo ambiente ha planteado y que reconoce como individuales?" (Mesa, 2005). Por lo anterior, se trata de un área enorme, donde las relaciones entre personas, el involucramiento y compromiso de los participantes en la prestación del servicio son elementos clave, y donde la producción y el consumo ocurren simultáneamente, convirtiendo el servicio en perecedero con pocas posibilidades de almacenamiento (Schneer, 1997).

La obra servucción (Eiglier \& Langeard, 1999) describe los elementos de un sistema de prestación de servicios (clientes, soporte físico, personal en contacto, servicio, organización interna y otros clientes), "por lo tanto vicio de calidad debe analizarse desde su concepción a la puesta en funcionamiento, con la diferencia que el cliente forma parte del sistema productivo" (Ferrano, 2008).

Un tipo especial de servicio, por la responsabilidad que le exige la sociedad, es el educativo, que por lo tanto requiere un diseño especial en sus esfuerzos de marketing, orientado de manera más social que comercial, basado en valores (Manes, 2005).

Basándose en las anteriores afirmaciones, la construcción del un plan de marketing, si bien sigue orientaciones generales para alcanzar los objetivos estratégicos (Vértice, 2007), debe abordarse con especial consideración en una institución educativa, de manera que corresponda a una planeación orientada adecuadamente y que contemple todas las variables necesarias para tomar decisiones que le permitan alcanzar el éxito (Luther, 2003).

\section{Análisis Estratégico}

\section{Análisis del Entorno}

A nivel de pregrado, entre las carreras ofrecidas por las distintas universidades se agrupan en Ingenierías, Ciencias de la Salud, Ciencias Económicas y Administrativas, Ciencias Básicas, Ciencias de la Educación, Ciencias, Bellas Artes, y Ciencias Jurídicas. En Barranquilla, la oferta educativa se observa en la tabla 1, encontrándose un comportamiento creciente en las matrículas, mientras que es estable en ingeniería, reflejándose un leve decrecimiento entre 2007 y 2009.

\subsection{Tendencias del Mercado}

La actividad económica actual, exige particularmente en el campo de la ingeniería, desafíos esenciales que responden a las necesidades de una población cada vez mayor. Estos desafíos se basan en cuatro importantes pilares: la sostenibilidad, la salud, la reducción de la vulnerabilidad y la calidad de vida.

Por tal razón, la academia funda el programa Ingeniería de Materiales, una rama de la ingeniería basada en las relaciones propiedades - estructura - procesamiento y diseña o proyecta la combinación de materiales para conseguir un conjunto predeterminado de propiedades.

En síntesis, las exigencias de la dinámica económica, exigen al mundo volcar su mirada al diseño, fabricación y comportamiento de todo tipo de componentes y estructuras, a la utilización tanto de materiales tradicionales como de nuevo diseño. En este sentido, la ingeniería de materiales se convierte en la base de 
los avances tecnológicos e innovadores que han transformado y transformaran la sociedad, en consecuencia, la demanda de profesionales con este perfil se incrementa.

\subsection{Análisis de Oferta}

La oferta de programas profesionales, de ingeniería y específicamente de ingeniería de materiales se observa en las tablas 3 y 4 :
El análisis de la tabla 3 muestra que a nivel nacional la matrícula en ingeniería representa alrededor del 23\% del total de matriculados en programas profesionales, en la cual la matrícula en ingeniería de materiales representa menos del 1\% (según las cifras obtenidas del Ministerio de Educación Nacional, se ofertan 3.136 programas profesionales, de los cuales 635 son de alguna ingeniería y sólo 5 son programas de ingeniería de materiales).

Tabla 1. Matrículas en programas profesionales en IES de Barranquilla totales y en ingeniería 2007 - 2010

\begin{tabular}{|c|c|c|c|c|c|c|c|c|}
\hline \multirow{2}{*}{ Institución } & \multicolumn{2}{|c|}{2007} & \multicolumn{2}{|c|}{2008} & \multicolumn{2}{|c|}{2009} & \multicolumn{2}{|c|}{2010} \\
\hline & Matriculas & Ing. & Matriculas & Ing. & Matriculas & Ing. & Matriculas & Ing. \\
\hline Corporación Universitaria de la Costa & 5.138 & 1.951 & 4.731 & 2.086 & 5.801 & 2.339 & 6.496 & 2.653 \\
\hline $\begin{array}{l}\text { Corporación Universitaria Empre- } \\
\text { sarial de Salamanca }\end{array}$ & 438 & 0 & 511 & 0 & 422 & 0 & 374 & 0 \\
\hline Universidad Autónoma del Caribe & 8.538 & 3.153 & 9.120 & 3.049 & 9.280 & 2.913 & 9.408 & 2.835 \\
\hline Universidad del Atlántico & 12.394 & 1.712 & 12.190 & 1.623 & 12.610 & 1.634 & 14.047 & 2.128 \\
\hline Universidad del Norte & 8.923 & 3.156 & 9.177 & 3.050 & 9.194 & 2.938 & 7.780 & 2.310 \\
\hline Universidad Metropolitana & 4.684 & 0 & 3.466 & 0 & 4.544 & 0 & 4.386 & 0 \\
\hline Universidad Simón Bolívar & 8.541 & 1.661 & 9.044 & 1.732 & 9.352 & 1.628 & 10.772 & 1.535 \\
\hline Totales & 48.656 & 11.633 & 48.239 & 11.540 & 51.203 & 11.452 & 53.263 & 11.461 \\
\hline
\end{tabular}

Fuente: www.mineducacion.gov.co

Tabla 3. Total matrículas a nivel nacional, matrículas en ingeniería e ingeniería de materiales 2008-2011

\begin{tabular}{|c|c|c|c|c|c|c|c|c|}
\hline MATRICULAS & 2008-1 & 2008-2 & 2009-1 & 2009-2 & 2010-1 & 2010-2 & 2011-1 & 2011-2 \\
\hline TOTAL NACIONALES & $1,241,881$ & $1,215,881$ & $1,336,612$ & $1,336,880$ & $1,448,021$ & $1,428,938$ & $1,554,362$ & $1,942,485$ \\
\hline INGENIERIA & 227,737 & 228,598 & 239,771 & 238,807 & 251,922 & 244,452 & 273,764 & 271,707 \\
\hline NGENIERIA DE MATERIALES & 762 & 806 & 791 & 767 & 689 & 304 & 797 & 719 \\
\hline
\end{tabular}

Fuente: www.mineducación.gov.co

Tabla 4. Matrículas en Ingeniería de Materiales 2008 - 2011

\begin{tabular}{|c|c|c|c|c|c|c|c|c|}
\hline IES & 2008-1 & 2008-2 & 2009-1 & 2009-2 & 2010-1 & 2010-2 & 2011-1 & 2011-2 \\
\hline UNIVERSIDAD DE ANTIOQUIA & 420 & 447 & 481 & 452 & 418 & 0 & 470 & 438 \\
\hline UNIVERSIDAD DEL VALLE & 342 & 345 & 296 & 289 & 250 & 277 & 293 & 246 \\
\hline UNIVERSIDAD AUTONOMA DEL CARIBE & 0 & 0 & 0 & 0 & 0 & 0 & 8 & 7 \\
\hline UNIVERSIDAD SANTO TOMAS & 0 & 0 & 0 & 0 & 0 & 0 & 0 & 0 \\
\hline UNIVERSIDAD DE SAN BUENAVENTURA & 0 & 14 & 14 & 26 & 18 & 26 & 25 & 27 \\
\hline UNIVERSIDAD ANTONIO NARIÑO & 0 & 0 & 0 & 0 & 3 & 1 & 1 & 1 \\
\hline TOTAL & 762 & 806 & 791 & 767 & 689 & 304 & 797 & 719 \\
\hline
\end{tabular}

Fuente: www.mineducación.gov.co 
Las instituciones que ofrecen Ingeniería de Materiales en Colombia son la Universidad del Valle, Universidad de Antioquia, Universidad de San Buenaventura, Universidad Antonio Nariño y Universidad Autónoma del Caribe (tabla 4), siendo las dos universidades públicas las que absorben la mayor parte de la matrícula, toda vez que la creación del programa en las últimas tres es reciente. Ello da una explicación de la baja matrícula en el programa.

\subsection{Análisis de Demanda}

De acuerdo al Informe de Calidad de Vida de Barranquilla, realizado por Barranquilla Como Vamos, para el 2010 existen en la ciudad 632 establecimientos educativos, con un total de 306.608 estudiantes matriculados, de los cuales 142.109 se encuentran en secundaria y media. Para ese año 16.596 estudiantes presentaron las Pruebas Saber 11.

Por otra parte, como se expone en el Diagnóstico de Competitividad del Atlántico 2011 elaborado por la Cámara de Comercio de Barranquilla, se evidencia que el $75,6 \%$ de los matriculados en nivel de educación superior elige formación universitaria, específicamente en carreras afines con las matemáticas, entre ellas las ingenierías y administración. Por tal motivo, los programas académicos con mayor número de registro calificado y acreditación de alta calidad son Ingeniería, Economía, Administración y afines.

Gráfico 1. Distribución de matrículas por nivel de formación en el Atlántico.

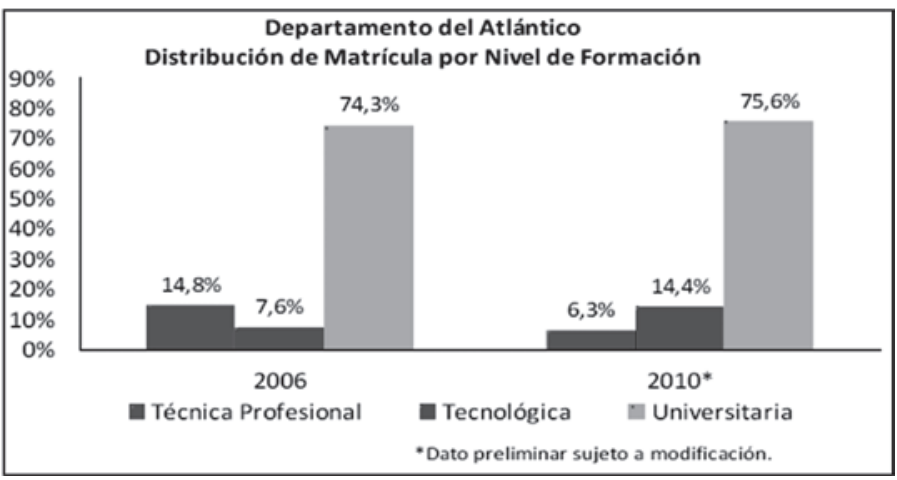

Fuente: Diagnóstico de competitividad del Atlántico 2011. Cámara de Comercio de Barranquilla.

\subsection{Investigación en Colegios}

Para el análisis de la percepción de los estudiantes de media vocacional de los colegios del Atlántico, se seleccionaron colegios en las cinco localidades de Barranquilla, para la aplicación de un instrumento de evaluación, con el apoyo de estudiantes de sexto semestre de Ingeniería Industrial. Cabe anotar que algunos estudiantes aplicaron encuestas en los municipios de Baranoa y Piojó.

Finalmente, de los 42 colegios visitados, algunos no atendieron la solicitud por múltiples razones, siendo encuestados en total 28 colegios en Barranquilla, Soledad, Baranoa y Piojó ${ }^{1}$. Los colegios que accedieron a la aplicación del instrumento se presentan en la tabla 7:

Tabla 7. Colegios donde se aplicó satisfactoriamente la encuesta.

\begin{tabular}{|c|c|c|}
\hline Nombre del Colegio & $\begin{array}{c}\text { Tipo de } \\
\text { institución }\end{array}$ & Municipio \\
\hline Colegio Colón & Privado & Barranquilla \\
\hline La Salle & Mixto & Barranquilla \\
\hline Colegio Domingo Sabio & Público & Barranquilla \\
\hline Instituto Ariano & Privado & Barranquilla \\
\hline Biffi La Salle & Privado & Barranquilla \\
\hline Colegio la Medalla Milagrosa & Privado & Barranquilla \\
\hline Instituto Pestalozzi & Público & Barranquilla \\
\hline ITIDA & Público & Soledd \\
\hline INEM & Público & Soledad \\
\hline Instituto San José & Mixto & Barranquilla \\
\hline Instituto Nueva Granada & Público & Barranquilla \\
\hline Colegio Distrital la Salle & Público & Barranquilla \\
\hline Colegio Nazareth Olaya & Público & Barranquilla \\
\hline Barranquilla para señoritas & Público & Barranquilla \\
\hline La Sagrada familia & Privado & Barranquilla \\
\hline Colegio Experimental & Público & Barranquilla \\
\hline Instituto Alexander Von Humboldt & Público & Barranquilla \\
\hline Centro Educativo la Sabiduría & Privado & Soledad \\
\hline Colegio San Gabriel & Público & Soledad \\
\hline Colegio cooperativa de Avianca & Privado & Soledad \\
\hline Colegio Francisco José de Caldas & Público & Soledad \\
\hline IED Simón Bolivar & Público & Soledad \\
\hline Colegio San Miguel del Rosario & Privado & Barranquilla \\
\hline José Eusebio Caro & Público & Barranquilla \\
\hline Dolores María Ucros & Privado & Soledad \\
\hline IDETEC & Privado & Soledad \\
\hline Tres colegios en Baranoa y Piojó & Público & \\
\hline
\end{tabular}

Fuente: Investigación realizada con apoyo de estudiantes.

Los resultados generales del instrumento aplicado se presentan a continuación.

- Fueron encuestados en total 885 estudiantes, de los cuales 521 fueron hombres y 364 mujeres (59\% y 41\% respectivamente)

\footnotetext{
Debe anotarse que los colegios fuera de Barranquilla y Soledad fueron escogidos por la ubicación de los estudiantes de sexto semestre de Ingeniería Industrial.
} 
- La edad promedio de los estudiantes encuestados fue 16,52 años, con una desviación estándar de 0,98 años.

- $\quad$ El rendimiento de los estudiantes encuestados es medio bajo en todas las áreas, lo que podría indica que ingresan con ciertas deficiencias a los programas de educación superior.

- Lo que más les interesa a los estudiantes conocer al momento de presentarles un programa académico es la proyección laboral que pueda tener, seguido del plan de estudios (pensum) del mismo.

- La mayor evaluación de percepción la obtuvo la Universidad del Norte, con un puntaje ponderado de 4.54 , seguida por la Universidad Autónoma del Caribe con 4,00 y la Universidad del Atlántico con 3,88. La institución con peor evaluación fue la CUC.

- Los estudiantes reflejan una gran autonomía al momento de seleccionar la carrera a estudiar, siendo la proyección laboral el elemento que más influye en su decisión (65\%), seguido de la proyección laboral (26\%).

- En su gran mayoría los estudiantes aspiran a estudiar un programa profesional (80\%). Una minoría insignificante no desea cursar estudios superiores.

- El mayor interés para estudiar un programa profesional se encuentra en el área de ingenierías (47\%), seguido de ciencias administrativas, económicas y contables (19\%).

- $\quad$ El 20\% de los estudiantes no tiene claro el perfil de la carrera que desea estudiar.

- $\quad$ El 69\% de los estudiantes tendría interés en conocer sobre una carrera nueva.

- Sólo el 20\% de los encuestados manifestó conocer el programa de ingeniería de materiales, lo que muestra que aún es muy poco el reconocimiento adquirido por el programa.

- El medio por el cual se han enterado de la existencia del programa con mayor puntaje es a través de información suministrada por familiares / amigos.

- Los estudiantes se informan sobre la oferta educativa principalmente a través de Internet, seguido de referencias de familiares o amigos y la televisión.

En términos generales se observó gran receptividad por parte de los estudiantes para informarse sobre el programa de ingeniería de materiales, pese al escaso conocimiento que se reflejó acerca del mismo.

\section{Análisis Interno}

La Universidad Autónoma del Caribe, fue fundada en 1967 por el doctor Mario Ceballos Araujo. Actualmente se encuentra ubicada en la Calle 90 con Carrera 46, una de las principales arterias de la ciudad de Barranquilla. Con más de cuarenta y cinco años de experiencia, ofrece alrededor de veintinueve (29) programas de pregrado, entre ellos encontramos la facultad de ingeniería, la cual incluye el programa de Ingeniería de Materiales.

En lo referente a su infraestructura física, cuenta con 27.000 $\mathrm{mt}^{2}$, siete bloques académicos, 107 aulas teóricas, 12 aulas de talleres y laboratorios, biblioteca y áreas de acceso adecuadas para el desarrollo y la formación profesional de los estudiantes. Además cuenta con un equipo docente altamente calificado, de reconocida trayectoria profesional e idoneidad académica.

\subsection{Resultados encuesta cliente interno}

Para ahondar en el análisis interno, se aplicó una encuesta entre los estudiantes del programa (a quienes se denominó cliente interno).

- Los resultados se presentan a continuación.

- Fueron encuestados estudiantes de semestres impares en su mayoría (47\% III semestre y $47 \%$ de I semestre), lo cual tiene su explicación en el hecho de que el primer semestre de cada año se cuenta con mayor número de matriculados en primer semestre, pues la mayoría de nuestros estudiantes provienen de colegios calendario A.

- La mayoría de los estudiantes del programa son hombres, sólo el $24 \%$ son mujeres.

- Los medios por los cuales los estudiantes que cursan Ingeniería de materiales se han enterado de la existencia del programa son Internet (39\%) y referencias familiares o de amigos (26\%). Esto refleja la misma tendencia de la encuesta externa aplicada.

- Los estudiantes del programa son personas jóvenes, con edades en su mayoría entre los 17 y 19 años.

- De acuerdo con los estudiantes encuestados, lo que más atrajo para decidirse a estudiar el programa fue su propuesta innovadora.

\section{Acciones propuestas de Marketing}

A continuación se presentarán algunas acciones diseñadas para difundir información sobre el programa de Ingeniería de Materiales, con el fin de incrementar su reconocimiento en la población y de esta manera aumentar la población estudiantil.

Cabe anotar que las estrategias estarán centradas principalmente en la variable promoción, toda vez que las características de producto y precio están fijadas institucionalmente, y que las particularidades del servicio que se ofrece hacen innecesario el despliegue de la variable distribución. Sin embargo, se presenta a continuación una breve descripción de cada variable, excluyendo la última en mención.

\subsection{Producto}

La Universidad Autónoma del Caribe define la Ingeniería de Materiales como una profesión orientada a la obtención, procesamiento, aplicación y evaluación de materiales (metales, cerámicos, polímeros y compuestos), con el fin de contribuir al desarrollo tecnológico y social, teniendo en cuenta la protección del medio ambiente. 


\section{Análisis DOFA}

Gráfico 2. Matriz DOFA del programa de ingeniería de materiales de la Universidad Autónoma del Caribe.

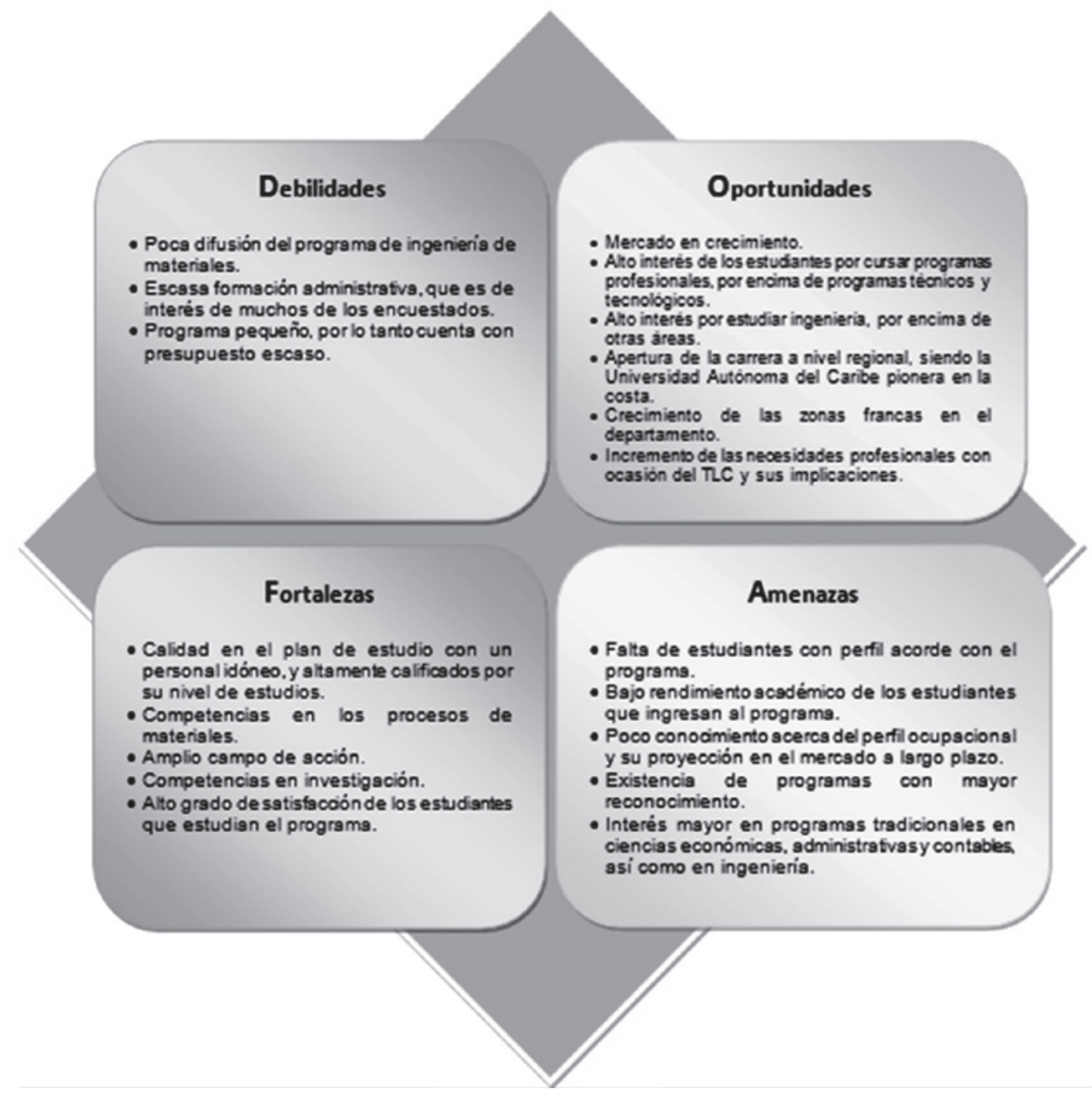

Fuente: Análisis de los autores a partir de la información recolectada.

\subsubsection{Campos de desempeño profesional}

Como la industria de nuestra Región Caribe Colombiana es variada, el ingeniero de materiales tiene actuación en industrias como la siderúrgica, metalmecánica, metalúrgica, química y petroquímica, plásticos y caucho, madera, cemento, cerámica y refractarios, vidrio, refrigeración, minería, industria naval, aeronáutica, automotriz, de servicios, asesorías, consultorías, investigación, entre otras.

\subsubsection{Competencias profesionales}

- Desarrollar procesos de fabricación de materiales que contribuyan al aumento de la productividad y el mejoramiento de la calidad de los productos con criterios de seguridad, confiabilidad y desarrollo sostenible.
- Capacidad para la selección y especificación de materiales y sus técnicas de fabricación.

- Capacidad desarrollar interdisciplinariamente proyectos de ingeniería donde se creen o adapten tecnologías avanzadas para dar soluciones concretas e innovadoras a necesidades del entorno, en un marco de desarrollo sostenible.

- Capacidad para la investigación y desarrollo en el campo de los materiales metálicos, poliméricos, cerámicos y compuestos.

- Capacidad para valorar el impacto que las soluciones de ingeniería producen en el medio ambiente y en el contexto social global.

- Capacidad de manejo de herramientas computacionales para el control, simulación, automatización y diseño de equipos y materiales. 


\subsubsection{Líneas de énfasis}

- Procesamiento de materiales. Está relacionada con el desarrollo y procesamiento de materiales metálicos, poliméricos, cerámicos y compuestos, y se orienta hacia la optimización de los recursos para contribuir al desarrollo de materiales aplicables a la industria con consideraciones éticas y ambientales.

- Análisis y Control de Materiales. Esta línea se basa en la aplicación de ensayos y técnicas necesarias para la caracterización y estudio de los materiales, ajustadas a la normatividad actual bajo criterios de sostenibilidad y preservación del medio ambiente.

- Desarrollo de herramientas computacionales y simulación de problemas de ingeniería. El objetivo de esta área es simular y modelar procesos en ingeniería mediante el uso o desarrollo de software, al tiempo que se forma talento humano en esa área creando una cultura de conocimiento, divulgación y aplicación.

\subsection{Precio}

A continuación se relacionan los valores de derechos académicos en la Universidad Autónoma del Caribe:

Tabla 1. Valores de derechos académicos Universidad Autónoma del Caribe

\begin{tabular}{|l|l|}
\hline \multicolumn{1}{|c|}{ Ítem } & \multicolumn{1}{c|}{ Valor } \\
\hline Inscripción & $\$ 70.000$ \\
\hline Matrícula Ingeniería de materiales & $\$ 3.090 .000$ \\
\hline
\end{tabular}

Fuente: Secretaría General.

\subsection{Promoción}

A continuación se presentan las acciones propuestas para la promoción del programa, especificando el grado de desarrollo actual y requerido para su implementación.

\subsubsection{Juego de materiales}

Considerando que la mayoría de los estudiantes encuestados utiliza Internet para informarse sobre carreras profesionales, además del crecido interés por propuestas innovadoras, se propone el diseño de un juego didáctico ubicado en la página de la Universidad Autónoma del Caribe, donde mostraremos como podemos emplear la ingeniería de materiales mediante ejemplos y juegos. Así los que visiten la página podrán enterarse del programa y conocer un poco más de lo que se trata en esta carrera.

Los visitantes al juego deberán registrar sus datos personales de contacto antes de acceder al mismo, generándose así una base de datos a ser contactada por el departamento de marketing, con el fin de atraer nuevos prospectos al programa.

\subsubsection{Feria experimental de materiales}

Se propone la creación de una feria que contiene tres elementos fundamentalmente:

- Exposición de experimentos hechos por estudiantes de ingeniería de materiales, explicando todos los procesos que ocurren dentro del experimento, para qué sirven, los distintos tipos de materiales y sus propiedades.

- En paralelo, se realizará un recorrido por los laboratorios utilizados por el programa de Ingeniería de Materiales, explicando las utilidades de cada uno.

- Presentación de proyectos de los estudiantes invitados, mediante un modelo de concurso, con premios propuestos así: al primer puesto media beca; al segundo y al tercer puesto, $30 \%$ y $15 \%$ descuento en su matrícula, respectivamente.

La invitación a los estudiantes para que participen en la feria que se realizará en la Universidad Autónoma del Caribe, se llevará a cabo mediante visitas a los colegios, así como notas de prensa y divulgación por los medios institucionales (página web, espacios radio visuales) se hará durante el primer semestre del año (durante los meses de marzo a mayo), para dar a los grupos interesados en participar la oportunidad de diseñar sus proyectos con tiempo suficiente.

\subsubsection{Redes sociales}

Crear un perfil en las redes sociales, mostrando imágenes de trabajos realizados por la comunidad académica del programa, generando además interacción con cualquier persona interesada en el mismo. Esta estrategia busca interactuar con la población juvenil, para lo cual se propone sea seleccionado un grupo de estudiantes del programa, para que en representación del mismo y en coordinación con la dirección del programa administre las cuentas en cada red social. Además de lo anterior, cada estudiante que ingrese al programa será invitado a vincularse con el perfil de Ingeniería de Materiales. Los estudiantes selectos podrán ser cambiados o ratificados cada semestre, según criterio de la dirección del programa, y, en todo caso, recibirán un reconocimiento escrito por su aporte al desarrollo del mismo.

\subsubsection{Visitas a colegios en los municipios}

Para dar un mayor conocimiento de la carrera, se propone mantener las relaciones públicas, llegando directamente al mercado objetivo mediante visitas a los colegios, en los cuales se entreguen accesorios que identifique el programa de Ingeniería de Materiales de la Universidad (como bolígrafos y manillas con el logotipo de la Universidad y del programa de Ingeniería de Materiales), también entregar folletos con el plan de estudio y la proyección laboral de la carrera. La propuesta es que esta estrategia se mantenga sólo para colegios selectos, especialmente los ubicados en los municipios fuera del Área Metropolitana de Barranquilla. 


\subsubsection{Concurso anual de Ciencias e Ingeniería de Materiales.}

Desarrollar un concurso anual de ciencias e ingeniería de materiales para familiarizar a los estudiantes bachilleres con el programa, además de fortalecer las competencias científicas entre los jóvenes de la región. Este concurso estará dirigido a estudiantes de los tres últimos años de bachillerato. Como premio para los ganadores, se plantea otorgar becas para estudiar o dinero en efectivo.

\subsubsection{Congreso de Ingeniería de materiales}

Se propone la organización de un congreso en el que se expongan temáticas de relevancia para la Ingeniería de Materiales y que además permita a la comunidad externa conocer sobre su aplicación. Considerando lo anterior, este congreso será abierto a los estudiantes de ingenierías afines, estudiantes de educación superior interesados en el tema, pero también a estudiantes de colegios (se enviará invitación especial a los colegios que tienen énfasis en ciencias), así como empleados de empresas que tengan relación con la profesión.

Los objetivos del congreso serán incrementar la interacción de la comunidad en general con la Ingeniería de Materiales, y lograr la difusión del conocimiento en temas de gran interés para la profesión.

\subsubsection{Alianzas con colegios seleccionados}

Los resultados de las encuestas aplicadas a colegios arrojaron los siguientes resultados, en virtud de los cuales se indica en la última columna si el colegio tiene un perfil aceptable o no para establecer una alianza que fortalezca el programa de ingeniería de materiales:

La tabla 7 muestra los colegios que tienen un perfil adecuado para establecer relaciones cercanas con ellos, basado en tres criterios: rendimiento académico e interés por una nueva carrera (columna 1), colegios con énfasis en ciencias o afines (columna 2) y colegios de procedencia de los estudiantes actuales del programa (columna 3). Nótese que algunos colegios aparecen en ambas columnas 2 y 3.

\subsubsection{Patrocinio de juego en La ruta del saber}

Se propone aprovechar la coyuntura que representa el programa "La ruta del saber" que organiza la Universidad y que se presenta en el canal regional Telecaribe. Este programa representa un alto potencial para incrementar el reconocimiento del programa gracias a la cantidad de jóvenes estudiantes de colegio que lo ven, además de los participantes en cada emisión. Considerando lo anterior, se diseñará un juego para ser aplicado en las transmisiones, relacionado con el saber de la Ingeniería de Materiales y auspiciado por el programa. El diseño se hará en conjunto con los estudiantes de Ingeniería de la Universidad Autónoma del Caribe.

\subsection{Cronograma y presupuesto}

A continuación se presenta el cronograma y presupuesto del plan de promoción propuesto. Debe anotarse que los tiempos planteados corresponden a la ejecución de la actividad según lo indicado en cada uno de los numerales 4.3 .1 a 4.3 .8 que, sin embargo, pueden ser susceptibles de modificarse considerando los intereses del programa y de la Universidad.

Tabla 7. Colegios con perfil para contactar y establecer alianzas

\begin{tabular}{|l|l|l|}
\hline \multicolumn{1}{|c|}{$\begin{array}{c}\text { Rendimiento } \\
\text { académico e interés }\end{array}$} & \multicolumn{1}{|c|}{ Énfasis en ciencias o afines } & \multicolumn{1}{c|}{ Procedencia de estudiantes } \\
\hline INEM & Centro Docente Nuestra Señora del Buen Consejo & Colegio Nuestra Señora del Buen Consejo \\
\hline San Miguel del Rosario & Colegio Biffi la Salle & Colegio Biffi la Salle \\
\hline Pestalozzi & Colegio Colón & Colegio Colón \\
\hline San Gabriel & Colegio Nuestra Señora de Lourdes & Colegio José Eusebio Caro \\
\hline Colegio Nueva Granada & Colegio Nuestra Señora del Buen Consejo & Centro Social Don Bosco \\
\hline Distrital La Salle & Colegio Nuestra Señora del Carmen & Colegio Americano \\
\hline Simón Bolívar & Escuela Mixta San Clemente & Instituto San José \\
\hline & $\begin{array}{l}\text { Fundación Educativa Instituto Experimental del } \\
\text { Atlántico José Celestino Mutis }\end{array}$ & Colegio María Inmaculada \\
\hline & $\begin{array}{l}\text { Institución Educativa de María Auxiliadora (co- } \\
\text { legio de María Auxiliadora) }\end{array}$ & Colegio de la Costa \\
\hline & Instituto la Salle & \\
\hline & Liceo de Cervantes & \\
\hline
\end{tabular}

Fuente: Investigación realizada por los autores, con apoyo de estudiantes de sexto semestre de ingeniería industrial 
Tabla 8. Cronograma de actividades por estrategia propuesta

\begin{tabular}{|c|c|c|c|c|c|c|c|c|c|c|c|c|c|c|c|c|c|}
\hline \multirow{2}{*}{ ESTRATEGIA } & \multirow{2}{*}{ ACTIVIDADES } & \multicolumn{8}{|c|}{2013} & \multicolumn{8}{|c|}{2014} \\
\hline & & A & $S$ & $\mathrm{O}$ & $\mathrm{N}$ & $\mathrm{D}$ & E & $\mathrm{F}$ & M & A & M & $\mathrm{J}$ & $\mathrm{J}$ & A & $S$ & 0 & $\mathrm{~N}$ \\
\hline \multirow{3}{*}{ Juego de materiales } & Diseño del juego & & & & & & & & & & & & & & & & \\
\hline & Prueba del juego & & & & & & & & & & & & & & & & \\
\hline & Lanzamiento del juego & & & & & & & & & & & & & & & & \\
\hline \multirow{3}{*}{$\begin{array}{l}\text { Feria experimen-tal } \\
\text { de materiales }\end{array}$} & Promoción & & & & & & & & & & & & & & & & \\
\hline & Inscripciones & & & & & & & & & & & & & & & & \\
\hline & Ejecución & & & & & & & & & & & & & & & & \\
\hline \multirow{2}{*}{ Redes sociales } & Crear perfil & & & & & & & & & & & & & & & & \\
\hline & Administrar información & & & & & & & & & & & & & & & & \\
\hline \multirow{2}{*}{ Visitas a colegios } & Organizar agenda & & & & & & & & & & & & & & & & \\
\hline & Visitas a colegios & & & & & & & & & & & & & & & & \\
\hline \multirow{6}{*}{$\begin{array}{l}\text { Concurso anual de } \\
\text { ciencias e ingeniería } \\
\text { de materiales }\end{array}$} & Divulgación & & & & & & & & & & & & & & & & \\
\hline & Inscripción & & & & & & & & & & & & & & & & \\
\hline & Preselección & & & & & & & & & & & & & & & & \\
\hline & Preparación & & & & & & & & & & & & & & & & \\
\hline & Final & & & & & & & & & & & & & & & & \\
\hline & Publicación de resultados & & & & & & & & & & & & & & & & \\
\hline \multirow{4}{*}{$\begin{array}{l}\text { Congreso de } \\
\text { ingeniería de } \\
\text { materiales }\end{array}$} & Preparación & & & & & & & & & & & & & & & & \\
\hline & Divulgación & & & & & & & & & & & & & & & & \\
\hline & Inscripción & & & & & & & & & & & & & & & & \\
\hline & Ejecución & & & & & & & & & & & & & & & & \\
\hline \multirow{2}{*}{ Alianzas con colegios } & Visitas a colegios & & & & & & & & & & & & & & & & \\
\hline & Convenios con colegios & & & & & & & & & & & & & & & & \\
\hline \multirow{3}{*}{$\begin{array}{l}\text { Patrocinio de juego en } \\
\text { La ruta del saber }\end{array}$} & Diseño & & & & & & & & & & & & & & & & \\
\hline & Prueba y ajuste & & & & & & & & & & & & & & & & \\
\hline & Implementación & & & & & & & & & & & & & & & & \\
\hline
\end{tabular}

Fuente: Autores

Tabla 9. Resumen de costos de estrategias

\begin{tabular}{|l|r|}
\hline \multicolumn{1}{|c|}{ ESTRATEGIA } & \multicolumn{1}{c|}{ COSTO } \\
\hline Juego de materiales & $\$ 4.400 .000$ \\
\hline Feria experimental de materiales & $\$ 9.160 .000$ \\
\hline Redes sociales & $\$ 0$ \\
\hline Visitas a colegios & $\$ 2.228 .700$ \\
\hline Concurso anual de ciencias e ingeniería de materiales & $\$ 11.895 .000$ \\
\hline Congreso de ingeniería de materiales & $\$ 12.130 .000$ \\
\hline Alianzas con colegios & $\$ 504.000$ \\
\hline Patrocinio de juego en La ruta del saber & $\$ 0$ \\
\hline Sub total costo del plan & $\$ 40.317 .700$ \\
\hline Premios a los ganadores (estimado en descuentos) & $\$ 30.910 .000$ \\
\hline & $\$ 71.227 .700$ \\
\hline
\end{tabular}

Fuente: Autores 
Tabla 10. Ingresos generados por las estrategias

\begin{tabular}{|l|r|}
\hline \multicolumn{1}{|c|}{ ESTRATEGIA } & ING. TOTAL \\
\hline Juego de materiales & $\$ 0$ \\
\hline Feria experimental de materiales & $\$ 0$ \\
\hline Redes sociales & $\$$ \\
\hline Visitas a colegios & $\$ 0$ \\
\hline Concurso anual de ciencias e ingeniería de materiales & $\$ 8.000 .000$ \\
\hline Congreso de ingeniería de materiales & $\$ 6.720 .000$ \\
\hline Alianzas con colegios & $\$ 0$ \\
\hline Patrocinio de juego en La ruta del saber & $\$ 0$ \\
\hline Subtotal ingresos generados por el plan & $\$ 14.720 .000$ \\
\hline $\begin{array}{l}\text { Ingreso por incremento esperado en población es- } \\
\text { tudiantil }\end{array}$ & $\$ 94.830 .000$ \\
\hline
\end{tabular}

Fuente: Autores

Considerando que el plan propuesto debe ser ejecutado en su mayoría durante el año 2014, los ingresos generados por el mismo se reflejarán en el año 2015. Sin embargo, las cifras de inscripción y matrículas aplicadas en la proyección mantuvieron los valores del periodo actual (2013-02). Así mismo, sólo se consideran generados por el plan, los ingresos correspondientes a matrículas en primer semestre, no se incluyen los ingresos de los siguientes semestres por cada estudiante, al contemplarlos como resultado de las estrategias académicas y de retención que ejecute el programa. Además de los costos contemplados en el plan, se estiman los siguientes requerimientos de personal de apoyo para la ejecución de cada una de las actividades. Cabe anotar que pueden ejecutar actividades en paralelo, por lo cual el total no coincide con la suma aritmética de los requerimientos de cada estrategia.

Tabla 11. Requerimientos de personal de apoyo para cada actividad.

\begin{tabular}{|l|c|c|}
\hline \multicolumn{1}{|c|}{ ESTRATEGIA } & Adm. & Estudiantes \\
\hline 1. Juego de materiales & 1 & 3 \\
\hline 2. Feria experimental de materiales & 1 & 4 \\
\hline 3. Redes sociales & 1 & 2 \\
\hline 4. Visitas a colegios & 1 & 4 \\
\hline $\begin{array}{l}\text { 5. Concurso anual de ciencias e ingeniería } \\
\text { de materiales }\end{array}$ & 1 & 4 \\
\hline 6. Congreso de ingeniería de materiales & 1 & 4 \\
\hline 7. Alianzas con colegios & 1 & 0 \\
\hline 8. Patrocinio de juego en La ruta del saber & 1 & 3 \\
\hline Total & 2 & 10 \\
\hline
\end{tabular}

Fuente: Autores
Finalmente, a continuación se resumen los ingresos y gastos generados por el plan propuesto, incluyendo la adquisición de un equipo de cómputo para llevar de manera organizada la información de actividades ejecutadas, así como la gestión de las redes sociales. Igualmente, se considera un rubro para administración del plan, correspondiente a gastos varios en los que incurrirán los estudiantes, así como bonos por su colaboración, que pueden ser representados en efectivo o en descuentos en las matrículas.

Tabla 12. Resumen de ingresos y gastos del plan

\begin{tabular}{|l|r|}
\hline \multicolumn{1}{|c|}{ CONCEPTO } & \multicolumn{1}{c|}{ MONTO } \\
\hline (+) Ingresos plan & $\$ 14.720 .000$ \\
\hline (-)Egresos plan & $\$ 40.317 .700$ \\
\hline (+) Ingresos por incremento de población & $\$ 94.830 .000$ \\
\hline (-) Premios a participantes & $\$ 30.910 .000$ \\
\hline (-) Administración del plan & $\$ 8.000 .000$ \\
\hline (-) Equipo de cómputo & $\$ 2.000 .000$ \\
\hline Generado & $\$ 28.322 .300$ \\
\hline
\end{tabular}

Fuente: Autores

\section{Recomendaciones}

El Plan de Mercadeo actual fue establecido tomando como referencia los resultados obtenidos de estudiantes de $11^{\circ}$ grado del área metropolitana de Barranquilla. Se sugiere que la Institución a través de su departamento de Marketing, realice el estudio para el resto del Departamento del Atlántico y la región, teniendo en cuenta los antecedentes que debe tener en cuanto a matriculados y colegios de procedencia de estudiantes de la Facultad de Ingeniería.

\section{Agradecimientos}

Se hace un especial agradecimiento a los estudiantes del programa de Ingeniería Industrial de la Universidad Autónoma del Caribe en el periodo 201201 de sexto semestre y en el periodo 201301 de décimo semestre, quienes ofrecieron valioso apoyo en la recolección de la información y diseño de las estrategias del plan propuesto.

\section{Bibliografía}

Azócar, C. (2012). Estudio de posicionamiento de las marcas Universidad Austral de Chile e Ingeniería Civil Industrial. Trabajo de titulación. Puerto Montt, Chile.

Ceballos, A., Arévalo, B., \& Giraldo, J. (2012). Plan de Marketing para incrementar el número de estudiantes en el Centro Educativo la Sabiduría de la Ciudad de Barranquilla. Escenarios, 29-39. 
Eiglier, P., \& Langeard, E. (1999). Servucción, el marketing de servicios. Madrid: Mc Graw Hill/Interamericana de España.

Ferrano, G. (2008). La servucción: Una herramienta para la gestión. XXVII Congreso argentino de profesores universitarios de costos (págs. 1-13). Tandil: Universidad Nacional del Nordeste.

Grande, I. (2005). Marketing de los servicios. Madrid, España: ESIC Editorial.

Grönroos, C. (1990). Marketing y gestión de servicios. Madrid: Díaz de Santos.

Guiltinan, J., Paul, G., Madden, T., \& de Barón, C. (1998). Gerencia de marketing: estrategias y programas. Bogotá: Mc Graw Hill.

Kotler, P. (2001). Dirección de marketing. Madrid, España: Prentice Hall.

Lovelock, C., \& Wirtz, J. (2011). Marketing de servicios. México: Pearson Educación.
Luther, W. (2003). El plan de mercadeo: cómo prepararlo y ponerlo en marcha. Bogotá: Grupo Editorial Norma.

Manes, J. (2005). Marketing para instituciones educativas. Buenos Aires: Ediciones Granica S A.

Mesa, J. (2005). Del Marketing de servicios al marketing relacional. Revista Colombiana de Marketing, 60-67.

Nacional, M. d. (s.f.). www.mineducacion.gov.co. Recuperado el 15 de Abril de 2013

Ries, A., \& Trout, J. (1999). La guerra de la mercadotecnia. México: Mc Graw Hill.

Schneer, M. (1997). Marketing de servicios profesionales: construyendo la práctica profesional. México: Ediciones Granica SA.

Velásquez, J., \& Caldas, S. (2003). Aplicación de los conjuntos de selección en la estrategia de mercadeo. Estudios gerenciales, 13-38.

Vértice, E. (2007). Plan de marketing. Málaga, España: Editorial Vértice. 